\title{
Point-of-Care Testing for Anaemia in Children Using Portable Haematocrit Meter: A Pilot Study from Southwest Nigeria and Implications for Developing Countries
}

\author{
Olatunya Oladele ${ }^{1}$, Ogundare Olatunde ${ }^{1}$, Olaleye Abiola ${ }^{2}$, Agaja Oyinkansola $^{1}$, \\ Omoniyi Evelyn" ${ }^{1}$, Adeyefa Babajide ${ }^{1}$, Oluwadiya Kehinde ${ }^{3}$, Oyelami Oyeku'
}

ABSTRACT

BACKGROUND: Prompt and accurate diagnosis is needed to prevent the untoward effects of anaemia on children. Although haematology analyzers are the gold standard for accurate measurement of haemoglobin or haematocrit for anaemia diagnosis, they are often out of the reach of most health facilities in resource-poor settings thus creating a care gap. We conducted this study to examine the agreement between a point-of-care device and haematology analyzer in determining the haematocrit levels in children and to determine its usefulness in diagnosing anaemia in resource-poor settings.

METHODS: EDTA blood samples collected from participants were processed to estimate their haematocrits using the two devices (Mindray BC-3600 haematology analyzer and Portable Mission Hb/Haemotocrit testing system). A pairwise t-test was used to compare the haematocrit (PCV) results from the automated haematology analyzer and the portable haematocrit meter. The agreement between the two sets of measurements was assessed using the Bland and Altman method where the mean, standard deviation and limit of agreement of paired results were calculated.

RESULTS: The intraclass and concordance correlation coefficients were 0.966 and 0.936 . Sensitivity and specificity were $97.85 \%$ and $94.51 \%$ respectively while the positive predictive and negative predictive values were $94.79 \%$ and $97.73 \%$. The Bland and Altman's limit of agreement was $-5.5-5.1$ with the mean difference being -0.20 and a non-ignificant variability between the two measurements $(p=0.506)$.

CONCLUSION: Haematocrit determined by the portable testing system is comparable to that determined by the haematology analyzer. We therefore recommend its use as a point-of-care device for determining haematocrit in resource-poor settings where haematology analyzers are not available.

KEYWORDS: Child health, Anaemia, Point-of-Care devices, Early diagnosis and treatment, Nigeria

DOI: http://dx.doi.org/10.4314/ejhs.v26i3.8

\section{INTRODUCTION}

Anaemia is a widespread public health problem that is highly prevalent among children and pregnant women and is associated with poor prognosis (1). It is estimated that about 300 million children have anaemia worldwide and most of them are in the developing countries. The sub-Saharan Africa has the highest proportions of those affected (1-3). This has been attributed to the high prevalence of malaria, dietary inadequacies, helminthiasis, bacterial infections, sickle cell diseases, Human Immunodeficient Virus (HIV) infection and increasing number of non-communicable diseases in these countries (1$6)$.

The presence of anaemia in children may lead to outright mortality if severe and not promptly detected or treated. In addition, chronic anaemia

\footnotetext{
${ }^{1}$ Department of Paediatrics, Ekiti State University/ Teaching Hospital, Ekiti State, Nigeria

${ }^{2}$ Achieving Health Nigeria Initiative, Abuja, Nigeria

${ }^{3}$ Department of Surgery, Ekiti State University, Ekiti State, Nigeria

Corresponding Author: Dr Olatunya Oladele Simeon, Email: ladeletunya@yahoo.com Tel: +2348038617705
} 
negatively impacts a child's cognition, growth and development, school performance, ability to fight infection and ability to live a productive adult life(1-3).

Anaemia is assessed either by measuring the haematocrit otherwise known as the Packed Cell Volume (PCV) or by measuring the haemoglobin (Hb) concentration of an individual $(2-3,7)$. Clinical and epidemiological studies use either of the two measurements to detect anaemia, and a three-fold conversion is commonly used to equate the two measures as follows : (haematocrit = haemoglobin concentration $x$ 3) (7). Several methods are routinely used to monitor an individual's haematocrit (PCV) or $\mathrm{Hb}$ levels. These include the use of haematology analyzers, haematocrit centrifuge, cyanmethemoglobin method, gravimetric copper sulphate method and colour code haemoglobin estimation (8-10). Although the haematology analyzers are the gold standards and very accurate, they are electricitydependent, expensive, and may be out of reach of many health facilities in resource-poor settings of most developing countries (8).The cyanmethemoglobin method is cheaper compared to the haematology analyzers but more laborious and time consuming (10). The Haematocrit centrifuge requires electricity to power which may make it unsuitable for analysis when there is power outage, a situation that may delay diagnosis or appropriate intervention and lead to increased morbidity and mortality. Both gravimetric copper sulphate and colour code haemoglobin estimation methods are fraught with high observer errors that impinge their accuracy (8-9).

In recognition of these limitations, efforts are being made to find suitable, cost-effective and yet accurate methods of measuring the haemoglobin and haematocrit (PCV) levels of patients (8-11). Recently, portable point-of-care devices have been developed and used for these purposes. These devices are portable, easy to use, require minimal laboratory procedures and give immediate results that are displayed on its screen (8,11-13). Although a few studies have investigated their use in the Nigerian setting, these were mostly in the adult population $(3,14-15)$. Hence, data to support and validate the use of these devices in children are lacking in Nigeria. We therefore conducted this study to generate data to support or refute the use and accuracy of a portable haemoglobin/haematocrit meter as a point-of-care device for haematocrit estimation in children seeking health care at a tertiary health care facility in Southwest Nigeria. The findings of the study could help raise awareness about whether or not such devices give accurate results in the paediatric population, and possibly make case for their use in paediatric patients in resource-poor settings to reduce delay in diagnosis and prevent complications.

The Mindray BC-3600 auto Hematology analyzer was used as the gold standard in this study. It has been proven to have excellent performance based on its high accuracy and precision relative to other automated haematology analyzers as per the Clinical and Laboratory Standard Institute (CLSI) criteria (16).

\section{PATIENTS AND METHODS}

Study setting: This was a prospective and hospital based study conducted at the paediatric emergency unit of the Ekiti State University Teaching Hospital (EKSUTH) Ado Ekiti, southwest Nigeria. The study was conducted between October and December 2014. EKSUTH is a tertiary public health facility providing health care to citizens of Ekiti State in the southwestern part of Nigeria. It serves as a referral centre for other hospitals within the state and other adjoining states like Osun, Ondo, Kwara and Kogi. The hospital is located in Ado Ekiti which doubles as both the headquarters of Ado Local Government Area and the state capital. The city is mainly inhabited by the Yorubas of the south-western part of Nigeria and has a population of about 308,621 inhabitants (17). The children emergency ward is one of the paediatric point-of-care units of the hospital. It receives emergency paediatric patients and is managed by six doctors and fifteen nurses on the average. These are made up of five paediatric registrars, three paediatric nurses, twelve nurse-midwives and a supervising consultant paediatrician.

Study participants and design: The study subjects were children aged between 6 and 59 months presenting with medical conditions requiring haematological investigations during their routine visit(s) to the paediatric emergency unit for consultations and treatments. Convenience sampling method was used to recruit participants and the number of study participants recruited met 
the general recommendation of not less than 50 samples/subjects as stipulated by Bland and Altman and other authors in methods comparison/validation study $(11,18)$. The study procedures were explained to the caregivers or parents of the participants, and those whose parents/caregivers gave consent to participate were consecutively recruited. Venous EDTA blood samples taken from patients for routine laboratory tests were used for the study, and no extra blood was collected. The venous blood collected from the participants was processed to estimate the haematocrit using two different methods: a point-of-care haematocrit and haemoglobin testing device (the Portable Mission $\mathrm{Hb} /$ haematocrit testing system) (19) and the automated haematology analyzer (Mindray BC 3600, China) (20). The residents in the unit performed the portable haematocrit meter testing. They were trained and acquired competence on the use of the portable haematocrit testing system based on the manufacturer's specifications before the commencement of the study. The laboratory staffs who were all blinded to the point-of-care results from the portable device, performed the laboratory tests for haematocrit on the same EDTA sample used by the residents using the automated haematology (Mindray BC 3600, China). All sample processing and analysis were done within 8hours of sample collection each day and according to manufacturer's specifications. The processing time for most samples was within 2 hours of collection. When this was not possible, the samples were kept in refrigerator at $4-8^{0} \mathrm{C}$ until analysis. The samples were equilibrated with the room temperature just before analysis.

\section{Portable Mission ${ }^{\circledR} \quad \mathrm{Hb} / \mathrm{Haematocrit}$ testing} system: The portable Mission testing system ${ }^{\circledR}$ (Acon biotech San Diego USA) (19) consists of a portable meter (reader) and disposable test strips containing reagent in a dry form for single purpose designed photometer. The device can be operated by either three 1.5 Volt alkaline batteries or an AC adaptor and costs 25,000 naira in Nigerian currency (approximately $\$ 125$ USA dollars) using the prevailing exchange rate (21). It comes with test strips stored in strips containers to maintain their integrity and shelf life. The device uses reflectance photometry principle and the reaction in the strip is a modified azide- methemoglobin reaction in which sodium deoxycholate hemolyses erythrocytes and haemoglobin is released. Sodium nitrite converts the released haemoglobin to methaemoglobin and the absorbance is measured at wavelengths of $525 \mathrm{~nm}$. The meter uses venous whole blood or whole blood anticoagulated with EDTA or heparin and or capillary blood for testing and the tests were performed as stated by the manufacturer (19). Briefly, a micropipette provided by the manufacturer was used to transfer $10 \mu \mathrm{l}$ of venous EDTA blood obtained from each participant for their routine haematological investigations at the point-of-care (the children emergency ward) by the attending residents to the point-of-care device with the strip in place after which the device automatically calculates/estimates and digitally display both the $\mathrm{Hb}$ and Haematocrit (PCV) within 15 seconds of performing the test.

Haematology Analyser (Hemajet BC 3600, Mindray China): The Mindray ${ }^{\circledR}$ BC 3600 (China) is an automated blood cell counter for diagnostic use in clinical laboratories. It is an automated haematology analyzer with a 3-part differentiation that analyses many haematologic parameters including $\mathrm{Hb}$ and haematocrit levels (20). The analyzer has been proven to provide accurate and reliable results $(20,22)$. The tests were performed according to manufacturer's recommendations.

Quality control: The functions of the portable $\mathrm{Hb} / \mathrm{Haematocrit}$ meter and the haematology analyzer were standardised daily based on the recommendations of the manufacturers $(19,20)$. Known results were used to test-run the instruments daily before they were used to process samples. They were only used each day if they passed the quality control test. The performance of the haematology analyzer was assessed for quality control each day before being used for the study by measuring the control material and comparing the results with a normal range of values given for the parameters using the reagents and the $\mathrm{BC}-3 \mathrm{D}$ three levels of control (low, normal and high) ran daily. If the results were outside the normal range provided by the manufacturer, the machine was recalibrated with the SC-CAL PLUS calibrator provided by the manufacturer being used. Both the portable testing meter and the haematology analyzer were kept and maintained according to 
manufacturer's' specifications. The personnel involved in both the point-of-care testing with the portable meter on the ward and the laboratory staffs processing the automated haematology analyzer were blinded to either results, and all samples were analysed on the day of collection within 8hours.

Statistical analysis: The data was entered into personal computer and analysed using SPSS Version 18. A pairwise t-test was used to compare the haematocrit $(\mathrm{PCV})$ results from the automated haematology analyzer and the portable haematocrit meter. Agreement between the test methods was assessed using the Bland and Altman method, where the mean, standard deviation and limit of agreement of paired results were calculated. Figures plotting difference against the average were graphed as recommended by Bland and Altman (23). The Intraclass correlation coefficient (ICC) was used to determine the within subject variability of measured haematocrit (PCV) while the concordance correlation coefficient (CCC) was used to measure the agreement between the two methods. The sensitivity, specificity, positive predictive value, negative predictive value and kappa values were calculated to assess the performance of the two methods in screening for anaemia. A cut-off value of $\mathrm{Hb}$ concentration $\leq 10 \mathrm{~g} / \mathrm{dL}(\mathrm{PCV} \leq 30 \%)$ was used to define anaemia as recommended for children aged 6 months to 5 years (3). the study. Written informed consent was obtained from parents/caregivers of participants. In addition, the results of the investigations were acted upon and those diagnosed with anaemia received appropriate treatments.

\section{RESULTS}

In all, 184 children participated in the study. They were made up of 117 ( $63.6 \%$ ) males and 67 $(36.4 \%)$ females. The point-of-care device PCV measurements ranged between 10 and 44 while the automated hematology analyzer measurements were between 6 and 45, and the overall mean difference between the two measurements was 0.20. Pairwise analysis revealed that the haematocrit $(\mathrm{PCV})$ determined by the laboratory method (haematology autoanalyzer) was not significantly different from that measured by the point-of-care device-the portable haematocrit Mission testing device $(-0.20,95 \%$ CI: -0.60 to $0.19, \mathrm{p}=0.303$ ).

Table 1: Summary statistics of measured haematocrits of the patients

\begin{tabular}{lclcc}
\hline Variable & Mean & Minimum & Maximum & SD \\
\hline Laboratory & 28.8 & 6 & 45 & 7. \\
Haematocrit & 3 & & & 62 \\
Portable device & 29.0 & 10 & 44 & 7. \\
Haematocrit & 3 & & & 52 \\
\hline
\end{tabular}

Ethical approval: The ethical committee of the Ekiti State University Teaching Hospital approved

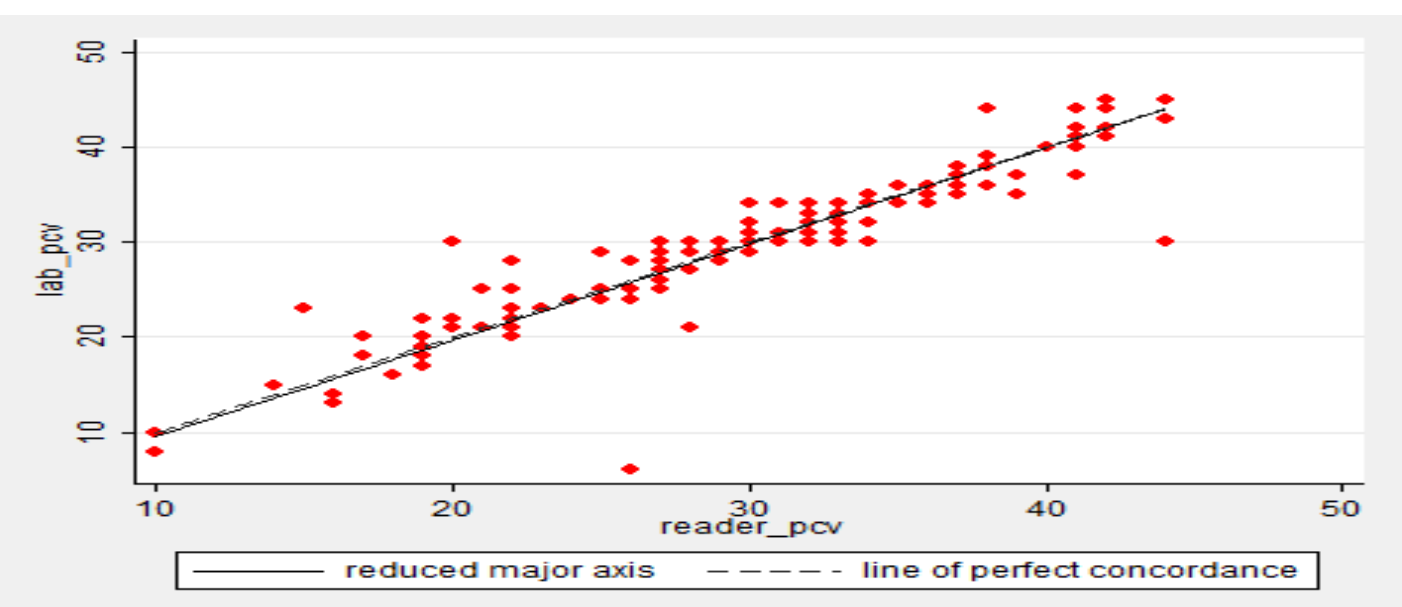

Figure 1: Normal plot of agreement between laboratory PCV and portable point-of-care PCV reader/testing device showing line of equality in which all measurements should lie if the two devices gave exactly the same readings on all the patients. As shown, most of the measurements align along the line of equality indicating good agreement between the measurements by the devices. 
There was near perfect concordance correlation between the haematocrit/ (PCV) determined by the laboratory and the portable haematocrit testing device methods (rho $=0.936$ and $\mathrm{p}<0.001$ ). Comparing the haematocrit results determined by the laboratory and the portable point-of-care haematocrit testing device methods, the Bland and Altman's limit of agreement was from -5.5 to 5.1. This indicates that the point-of-care testing device measured haematocrits may be 5.5 below or 5.1 above the measurements using the laboratory haematology analyzer with a mean difference of 0.20 , and a non-significant difference in variability between the two measurements $(\mathrm{p}=$ 0.506) (Figures $1 \& 2$ ). The intraclass correlation coeffiecient (ICC) was 0.966 (95\% confidence interval: $0.956-0.975)$ and the concordance correlation coefficient (CCC) was $0.936(95 \%$ confidence interval: $0.915-0.951)$. These results indicate a good agreement between the two measurements.

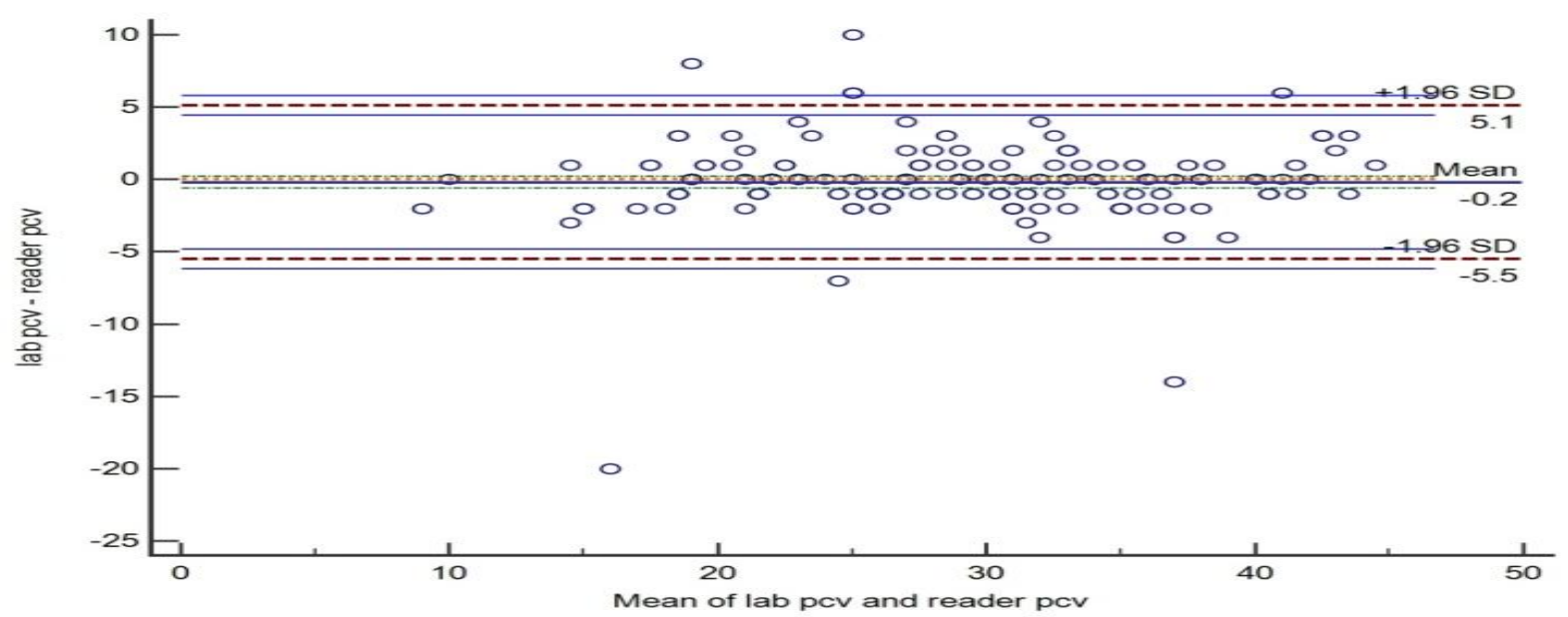

Figure 2: Bland and Altman plot for laboratory PCV and portable point-of-care haematocrit testing device NB: Figure 2 shows the plot of the Difference between laboratory PCV and portable point-of-care PCV testing device/reader (Lab PCV-Reader PCV) versus average of haematocrit (PCV) measured by portable point-of-care haematocrit reader and the hematology analyzer. Horizontal solid line represents mean difference: broken red lines represent $95 \%$ limits of agreement.

Lab_PCV = Haematocrit/PCV measured by the automated haematology analyser

Reader_PCV $=$ Haematocrit/PCV measured by the portable point-of-care haematocrit/PCV reader

The overall prevalence of anaemia was $50.5 \%$ using the automated haematology analyzer and $52.2 \%$ using the portable point-of-care haematocrit testing device. The kappa value was 0.924 which indicates a very good agreement. Using the automated haematology analyzer as the gold standard and the reference method, the sensitivity and specificity of the portable point-of-care haematocrit testing device in detecting anaemia were $97.85 \%$ (95CI: $92.45 \%$ to $99.74 \%$ ) and 94.51\% (95 CI: $87.64 \%$ to $98.19 \%$ ) respectively. The positive predictive value was $94.79 \%$ (95 CI: $88.26 \%$ to $98.29 \%$ ) while the negative predictive value was $97.73 \%$ (95CI: $92.03 \%$ to $99.72 \%$ ).

\section{DISCUSSION}

Anaemia affects millions of children worldwide and most of them are in the developing parts of the world especially in Asia and Africa (1-3). Haematocrit (PCV) and/or haemoglobin estimations/ measurements are frequently used to diagnose anaemia (7). In developing countries, most patients with anaemia present late in hospitals. Therefore, timely and accurate determination of these parameters is central to prompt diagnosis and institution of appropriate interventions. Although haematology analyzers are the gold standard for estimating haematocrits and haemoglobin, they are scarcely available in most resource-poor settings, and when available, they are usually placed in a central laboratory remote from patients. Ample time is often lost to transportation 
to the lab and processing of results. In addition, the autoanalyzers are often required to be powered by electricity which is usually not available as at when needed in most facilities in the developing countries including Nigeria. The costs of these analyzers are also prohibitive and may be too expensive for most health care facilities $(8-11,24)$.

The point-of-care haematocrit and $\mathrm{Hb}$ testing device requires very small blood volume $(10 \mu 1)$, does not require extensive laboratory procedures; is portable, inexpensive and easy to use; gives immediate, digitally displayed results at the bedside, and can function with the use of simple batteries without the need for electricity supply thus making it suitable for use in most resource-poor or rural settings. The intraclass correlation coefficient (ICC) and concordance correlation coefficient (CCC) are used to evaluate agreement between measurements and the values obtained in our study (0.966 and 0.936 respectively) are comparable or better than those reported by other studies that investigated the accuracies of point-of-care $\mathrm{Hb}$ or haematocrit testing devices $(8,11)$. In addition, the current study showed the point-of-care device to have both high sensitivity $(97.85 \%)$ and specificity $(94.51 \%)$ in detecting anaemia among the subjects. This observation is comparable to the findings by other authors who compared accuracies of point-ofcare testing devices in screening for anaemia among mixed subjects' population vis-à-viz the gold standard (automated haematology analyzers). They include Neufeld et al among adults and children (84\% and 97\%) (25), Zhou et al among pregnant women in china (94.9\% and $76.7 \%$ ) (11), as well as Sari et al in Indonesian mothers $(70.6 \%$ and $97.5 \%$ ) (10). These observations point to the reliability of the point-of-care device to measure haematocrits.

In Africa and most developing countries, getting prompt and accurate diagnosis is often a mirage due to inadequate diagnostic tools and skilled manpower. Modern high technology tools for diagnosis are prohibitively expensive. In addition, the tools often require experts to operate, and these experts are scarce in the region. Hence, the overhead costs of these factors has led to the services being too expensive for most patients (2627). For example, in a study from Kenya, nearly $15 \%$ of children with clinical histories indicative of malaria and anaemia could not have their haematocrit tested, and $25 \%$ of basic essential tools to facilitate diagnosis and treatment were lacking in 8 out of 14 surveyed hospitals (28). In this context, a reliable and low cost tool like the point-of-care device in this study would have improved the diagnosis of anaemia, prevented misdiagnosis or unwarranted interventions and reduced complications among the subjects. The landing cost of the portable device is 25,000 Nigerian Naira equivalent to US\$125.00 dollars (21), and the estimated cost per test, taking into considerations the cost of the strips and other consumables needed for testing, would have stood at 200 Naira (approximately \$1.00 USA dollar) per patient, were they to pay for the tests. This affirms the affordability of the device whose price is quite below the landing costs of most other point-of-care devices especially those used in more advanced settings to test haematocrit or haemoglobin which often need electricity to power $(13,24)$. In contrast, the device evaluated in this study does not depend solely on electricity to power it. It also uses three small 1.5 Volt alkaline batteries. This makes it and others with similar technology suitable for use in resource-poor settings where anaemia is a major comorbid condition and electricity supply is unreliable and inadequate $(2-3,7)$.

Although there are studies on the use of pointof-care devices in screening for anaemia among Nigerian patients $(3,14-15)$, only one was conducted on paediatric patients. However, the study did not validate the accuracy of the point-ofcare device and compare its measurements with a gold standard (3) as done in this study. Thus, this makes this study the first to validate a point of care device with a gold standard among Nigerian pediatric patients. It is interesting to note that the cost of conducting laboratory haematocrit testing at the study centre at the time of the study was between 200 and 350 naira (\$1.00 and \$1.75 US dollar) for paediatric and adults patients respectively. This is comparable to the estimated cost of testing using the point-of-care device if the patients were to pay for the tests. Given the timeliness, ease and accuracy of the results produced by the point-of-care device, it could be speculated that adopting devices with similar technology would easily obviate the problems of delayed diagnosis and intervention for children with suspected anaemia(1-3).

In addition, given the prevailing landing cost of haematocrit testing at the study time, the cost of 
testing with the device would have been more pocket friendly for the mixed patients population (paediatric and adult) seen in the hospital settings despite the price differentials enjoyed by the paediatric patients on some investigations at the study centre. This further reinforces the need for the use of point-of-care devices with similar technology and ease of testing for the screening and triage of patients at critical points of care such as children and adults' emergency points, antenatal clinics, and by extension, in field surveys for anaemia screening. However, the greatest potential beneficiaries of the technology may be the numerous primary health care centres in the country. These centres often do not have means of measuring haematocrit due to lack of appropriate personnel, equipment and electricity. Using pointof-care devices would improve the diagnoses, treatment and triaging of patients with anaemia.

Researchers have identified some problems associated with the use of point-of-care devices for anaemia to include results variability between venous and capillary samples. This is especially so if the tests are not performed as specified or the test strips or curvette are not maintained as recommended, and/or the device is not routinely checked to pass quality control test $(8,10-11)$. In addition, maintenance of these point-of-care devices requires some level of biomedical engineering backup which may not be routinely available in resource-poor settings $(8,10-11)$. Also, the costs of the strips and ensuring their regular supplies may hinder the use of point-of-care devices and their availability in resource-poor settings $(8,10)$. These problems can easily be prevented if the operators receive proper training before use, endeavour to pass the device through quality control checks routinely and strictly adhere to manufacturer's recommendations as done in this study. In addition, opening up channels to ensure continuous supply of materials needed for normal operations of such devices alongside proper fund turn around and or subsidy provision and supervision by government agencies may enhance the sustained use of the device in rural settings. In this study, we not only adhere to the manufacturer's recommendations on testing, quality control and device maintenance, we also ensure that there were no variability in the samples used for testing by conducting both the point of care testing and the laboratory testing with the same EDTA venous samples. This, perhaps, may explain why the haematocrit results (measurements) compare favourably with the standards. This is an indication that users of such devices need to adhere strictly to stated guidelines for the device in order to obtain optimal results.

In conclusion, venous haematocrit determination using the portable Mission haematocrit and $\mathrm{Hb}$ testing device have acceptable agreement with the measurements done using the laboratory haematology analyser. We therefore recommend further studies to evaluate this device and other point-of-care testing devices for anaemia screening among children and older patients. This study shows that the device has the potential to reduce the burden of delay in diagnosing anaemia and its untoward consequences among patients in resource-poor settings.

\section{ACKNOWLEDGEMENT}

The authors acknowledge with thanks the supports of the parents and caregivers for their cooperation during the study.

\section{REFERENCES}

1. Stevens GA, Finucane MM, De-Regil LM, Paciorek JC, Flaxman SR, Branca F. Global, regional, and national trends in haemoglobin concentration and prevalence of total and severe anaemia in children and pregnant and nonpregnant women for 1995-2011: a systematic analysis of population-representative data. Lancet Glob Health, 2013;1:e16-25.

2. Mathema S, Shrestha A. Prevalence and causes of anaemia in six to sixty months old children: A cross-sectional study at Kathmandu Medical College and Teaching Hospital. J Nepal Paediatr Soc, 2013;33(3):163-5.

3. Ughasoro MD, Emodi IJ, Okafor HU, Ibe BC. Prevalence and risk factors of anaemia in paediatric patients in South-East Nigeria. $S$ Afr $J$ CH, 2015;9:14-17 Doi:10.7196/SAJCH.760

4. Anumudu CI, Okafor CM, Ngwumohaike V, Afolabi KA, Nwuba RI, Nwagwu M. Epidemiological factors that promote the development of severe malaria anemia in children in Ibadan. Afr Health Sci, 2007;7(2):80. 
5. Callis JC, Phiri KS, Faragher EB et al. Severe anaemia in Malawi children. $N$ Eng $\mathrm{J} \mathrm{Med,}$ 2008;358(9):888-99.

6. Balarajan Y, Ramakrishman U, Ozaltin E, Shankar AH, Subramanian SV. Anemia in low and middle income countries. Lancet, 2011;378:2123-35.

7. Lee SJ, Steipneiwska K, Anstey N, Ashley E, Barnes K, Binh TQ. The relationship between the haemoglobin concentration and haematocrit in plasmodium falciparum malaria. Malaria Journal, 2008;7:149 Doi:10.1186/1475-2875.

8. Nkrumah B, Nguah SB, Sarpong N, Dekker D, Idriss A, May J. Hemoglobin estimation by the Hemocue portable haemoglobin photometer in a resource poor setting. BMC Clinical Pathology, 2011;11:5 Doi:10.1186/1472-6890-11-5.

9. Paddle JJ. Evaluation of the haemoglobin colour scale and comparison with the Hemocue haemoglobin assay. Bull World Health Org, 2002; 80:813-16.

10. Sari M, dePee S, Martini E, Herman S, Bloem MW, Yip R. Estimating the prevalence of anaemia a comparison of three methods. Bull World Health Org, 2001;79:506-11

11. Zhou X, Yan H, Xing Y, Dang S, Zhuoma B, Wang D. Evaluation of portable haemoglobin photometer in pregnant women in a high altitude area: a pilot study. BMC Public Health, 2009;9(1):228 Doi:10.1186/1471-2458-9-228.

12. Myers GJ, Browne J. Point of care haematocrit and haemoglobin in cardiac surgery: A review. Perfusion, 2007;22:179-83.

13. Patel KP, Hay GW, Cheteri MK, Holt DW. Haemoglobin test result variability and cost analysis of eight different analyzers during open heart surgery. J Extra Corpor Technology, 2007;39:10-7

14. Ivoke N, Eyo EE, Ivoke ON, Nwani CD, Odu $\mathrm{EC}$, Asogha $\mathrm{CN}$ et al. Anemia prevalence and associated factors among women attending antenatal clinic in south western Ebonyi State, Nigeria. Int $J$ Medicine and Medical Sci, 2013;46(1):1354-59.

15. Ojengbede OA, Okonkwo SN, Morhason-Bello IO. Comparative evaluation of haemoglobin estimation amongst pregnant women in Ibadan: Haemocue-B haemoglobin analyzer versus haemoglobin cyanide (standard) method as the gold standard. Afr $J$ Reproductive Health, 2008;12(2):153-9

16. Mindray BC-3600 auto-hematology analyzer. Available at www.accessdata.fda.gov Retrieved December 31, 2015.
17. Legal notice on publication of 2006 census final results if the Federal Republic of Nigeria, 2006 National Population Census official Gazette. Annexure A,B and C 2009; 96(2):B1-B42. Available at www.placng.org/legal/Notice on 2006 census final results. Retrieved February 20, 2015.

18. Bland JM, Altman DG. Measuring agreement in method comparison studies. Stat Methods Med Res, 1999;8(2):135-60.

19. Portable Mission $\mathrm{Hb}$ Haemoglobin testing system ACON laboratory San Diego USA. Available at www.aconlabs.com. Retrieved March 28,2015.

20. Mindray BC 3600 Haematology autoanalyzer China Mindray . Available at www.mindray.com. Retrieved March 28, 2015.

21. The Naira Exchange Rate. Available at http://www.xe.com Retrieved July 10, 2015.

22. Shu G, Lu H, Du H, Shi J, Wu. Evaluation of Mindray BC-3600 hematology analyzer in a University Hospital. Int $J$ Lab Hematology, 2013;35(1):61-9 Doi:10.1111/j.1751553x.2012.01464.x.Epub2012

23. Bland JM, Altman DG. Statistical methods for assessing agreement between two methods of clinical measurement. Lancet 1986;: 307-10.

24. Yang X, Piety NZ, Vignes SM, Benton MS, Kanter J, Shevkoplyas SS. Simple paper-based test for measuring blood haemoglobin concentration in resource-limited settings. Clin chem, 2013;59(10): Doi:10.1373/clinchem.2013.204701.

25. Neufeld L, Garcia-Guerra A, Sachez-Francia D, Newton -Sachez O, Ramiez-Villalobos MD, Rivera-Dommarco J. Hemoglobin measured by Haemocue and a reference method in venous and capillary blood: a validation study. Saudi Publica Mex, 2002;44(3):219-27.

26. Petti CA, Polage CR, Quinn TC, Roland AR, Sande MA. Laboratory medicine in Africa: a barrier to effective health care. Clin Infect Dis 2006;42:377-82

27. Bates I, Maitland K, Are laboratory services coming of age in sub-saharan Africa? Clin Infect Dis 2006;383.

28. English M, Esamai F, Wasuma A, Were F, Ogutu B, Wamae A et al. Delivery of paediatric care at the first-referral level in Kenya. Lancet, 2004;364:1622-29. 